\title{
Rainbow Matching in Edge-Colored Graphs
}

\author{
Timothy D. LeSaulnier*† Christopher Stocker* \\ Paul S. Wenger* $\quad$ Douglas B. West $*$
}

Submitted: Dec 29, 2009; Accepted: May 7, 2010; Published: May 14, 2010

Mathematics Subject Classification: 05C15, 05C35, 05C55, 05C70

\begin{abstract}
A rainbow subgraph of an edge-colored graph is a subgraph whose edges have distinct colors. The color degree of a vertex $v$ is the number of different colors on edges incident to $v$. Wang and Li conjectured that for $k \geqslant 4$, every edge-colored graph with minimum color degree at least $k$ contains a rainbow matching of size at least $\lceil k / 2\rceil$. We prove the slightly weaker statement that a rainbow matching of size at least $\lfloor k / 2\rfloor$ is guaranteed. We also give sufficient conditions for a rainbow matching of size at least $\lceil k / 2\rceil$ that fail to hold only for finitely many exceptions (for each odd $k$ ).
\end{abstract}

\section{Introduction}

Given a coloring of the edges of a graph, a rainbow matching is a matching whose edges have distinct colors. The study of rainbow matchings began with Ryser, who conjectured that every Latin square of odd order contains a Latin transversal [3]. An equivalent statement is that when $n$ is odd, every proper $n$-edge-coloring of the complete bipartite graph $K_{n, n}$ contains a rainbow perfect matching.

Wang and $\mathrm{Li}$ [4] studied rainbow matchings in arbitrary edge-colored graphs. We use the model of graphs without loops or multi-edges. The color degree of a vertex $v$ in an edge-colored graph $G$, written $\hat{d}_{G}(v)$, is the number of different colors on edges incident to $v$. The minimum color degree of $G$, denoted $\hat{\delta}(G)$, is $\min _{v \in V(G)} \hat{d}_{G}(v)$.

Wang and $\mathrm{Li}[4]$ proved that every edge-colored graph $G$ contains a rainbow matching of size at least $\left\lceil\frac{5 \hat{\delta}(G)-3}{12}\right\rceil$. They conjectured that a rainbow matching of size at least

*Department of Mathematics, University of Illinois, Urbana, IL 61801. Email addresses: tlesaul2@uiuc.edu, stocker2@uiuc.edu, pwenger2@uiuc.edu, west@math.uiuc.edu.

${ }^{\dagger}$ Contact author, partially supported by NSF grant DMS 08-38434 "EMSW21-MCTP: Research Experience for Graduate Students."

${ }^{\ddagger}$ Research supported by NSA grant H98230-10-1-0363. 
$\lceil\hat{\delta}(G) / 2\rceil$ can be guaranteed when $\hat{\delta}(G) \geqslant 4$. A properly 3-edge-colored complete graph with four vertices has no rainbow matching of size 2, but $\mathrm{Li}$ and $\mathrm{Xu}[2]$ proved the conjecture for all larger properly edge-colored complete graphs. Proper edge-colorings of complete graphs using the fewest colors show that the conjecture is sharp.

We strengthen the bound of Wang and Li for general edge-colored graphs, proving the conjecture when $\hat{\delta}(G)$ is even. When $\hat{\delta}(G)$ is odd, we obtain various sufficient conditions for a rainbow matching of size $\lceil\hat{\delta}(G) / 2\rceil$. Our results are the following:

Theorem 1.1. Any edge-colored graph $G$ has a rainbow matching of size at least $\lfloor\hat{\delta}(G) / 2\rfloor$.

Theorem 1.2. Each condition below guarantees that an edge-colored graph $G$ has a rainbow matching of size at least $\lceil\hat{\delta}(G) / 2\rceil$.

(a) $G$ contains more than $\frac{3(\hat{\delta}(G)-1)}{2}$ vertices.

(b) $G$ is triangle-free.

(c) $G$ is properly edge-colored, $G \neq K_{4}$, and $|V(G)| \neq \hat{\delta}(G)+2$.

Condition (a) in Theorem 1.2 implies that, for each odd $k$, only finitely many edge-colored graphs with minimum color degree $k$ can fail to have a rainbow matching of size $\lceil k / 2\rceil$, where an edge-coloring is viewed as a partition of the edge set. Condition (c) guarantees that failure for a properly edge-colored graph can occur only for $K_{4}$ or a graph obtained from $K_{k+2}$ by removing a matching.

A survey on rainbow matchings and other rainbow subgraphs appears in [1]. Subgraphs whose edges have distinct colors have also been called heterochromatic, polychromatic, or totally multicolored, but "rainbow" is the most common term.

\section{Notation and Tools}

Let $G$ be an $n$-vertex edge-colored graph other than $K_{4}$, and let $k=\hat{\delta}(G)$. If $n=k+1$, then $G$ is a properly edge-colored complete graph and has a rainbow matching of size $\lceil k / 2\rceil$, by the result of $\mathrm{Li}$ and $\mathrm{Xu}[2]$. Therefore, we may assume that $n \geqslant k+2$.

Let $M$ be a subgraph of $G$ whose edges form a largest rainbow matching, and let $c=k / 2-|E(M)|$. We may assume throughout that $c \geqslant 1 / 2$, since otherwise $G$ has a rainbow matching of size $\lceil k / 2\rceil$. Let $H$ be the subgraph induced by $V(G)-V(M)$, and let $p=|V(H)|$. Note that $p=n-(k-2 c)$. Since $n \geqslant k+2$, we conclude that $p \geqslant 2 c+2$.

Let $A$ be the spanning bipartite subgraph of $G$ whose edge set consists of all edges joining $V(M)$ and $V(H)$ (see Figure 1). We say that a vertex $v$ is incident to a color if some edge incident to $v$ has that color. A vertex $u \in V(M)$ is incident to at most $|V(M)|-1$ colors in the subgraph induced by $V(M)$, so $u$ is incident to at least $2 c+1$ colors in $A$. That is,

$$
\hat{d}_{A}(u) \geqslant 2 c+1 \text {. }
$$




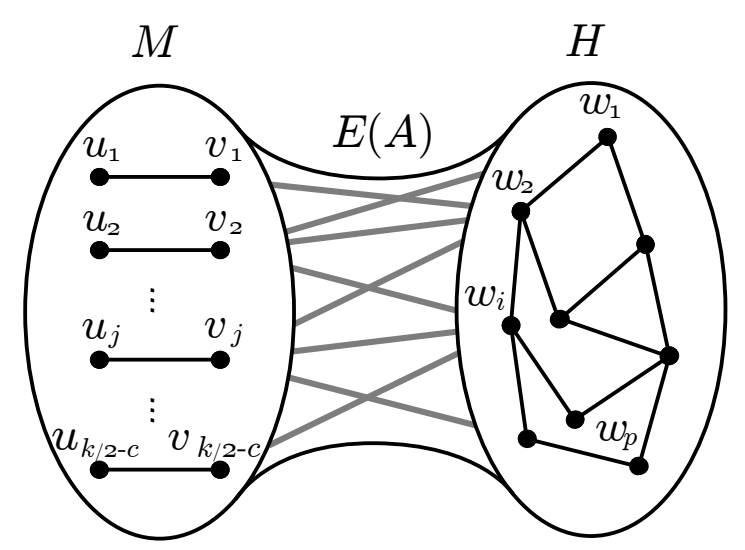

Figure 1: $V(M)$ and $V(H)$ partition $V(G)$.

We say that a color appearing in $G$ is free if it does not appear on an edge of $M$. Let $B$ denote the spanning subgraph of $A$ whose edges have free colors. We prove our results by summing the color degrees in $B$ of the vertices of $H$. We find upper and lower bounds for $\hat{d}_{B}(V(H))$, where $f(S)=\sum_{s \in S} f(s)$ when $f$ is defined on elements of $S$. These bounds will yield a contradiction when $c$ is too large, that is, when $M$ is too small.

There are only $k / 2-c$ non-free colors, so a vertex $w \in V(H)$ is incident to at least $k / 2+c$ free colors. By the maximality of $M$, no free color appears in $H$, so the free colors incident to $w$ occur on edges of $B$. That is, $\hat{d}_{B}(w) \geqslant k / 2+c$. Summing over $V(H)$ yields

$$
\hat{d}_{B}(V(H)) \geqslant p(k / 2+c) \text {. }
$$

Let the edges of $M$ be $u_{1} v_{1}, \ldots, u_{k / 2-c} v_{k / 2-c}$. For $1 \leqslant j \leqslant k / 2-c$, let $B_{j}$ be the subgraph of $B$ induced by $V(H) \cup\left\{u_{j}, v_{j}\right\}$. Note that $\hat{d}_{B_{j}}(w) \leqslant 2$ for $w \in V(H)$.

Lemma 2.1. If at least three vertices in $V(H)$ have positive color degree in $B_{j}$, then only one such vertex can have color degree 2 in $B_{j}$. Furthermore,

$$
\hat{d}_{B_{j}}(V(H)) \leqslant p+1 .
$$

Proof. Let $w_{1}, w_{2}$, and $w_{3}$ be vertices of $H$ such that $\hat{d}_{B_{j}}\left(w_{1}\right)=\hat{d}_{B_{j}}\left(w_{2}\right)=2$ and $\hat{d}_{B_{j}}\left(w_{3}\right) \geqslant$ 1. By symmetry, we may assume that $w_{3} v_{j} \in E\left(B_{j}\right)$. Maximality of $M$ requires $u_{j} w_{1}$ and $v_{j} w_{2}$ to have the same color. Since $\hat{d}_{B_{j}}\left(w_{2}\right)=2$, the color on $u_{j} w_{2}$ differs from this. Now $u_{j} w_{1}$ or $u_{j} w_{2}$ has a color different from $v_{j} w_{3}$, which yields a larger rainbow matching.

Now consider $\hat{d}_{B_{j}}(V(H))$. Since $p \geqslant 2 c+2$, we have $p \geqslant 3$. If $\hat{d}_{B_{j}}(V(H)) \geqslant p+2$, then $\hat{d}_{B}(w) \leqslant 2$ for all $w \in V(H)$ requires that there be three vertices as forbidden above.

For $p \geqslant 4$, the next lemma determines the structure of $B_{j}$ when $\hat{d}_{B_{j}}(V(H))=p+1$. Let $N_{G}(x)$ denote the neighborhood of a vertex $x$ in a graph $G$. 
Lemma 2.2. For $p \geqslant 4$, if $\hat{d}_{B_{j}}(V(H))=p+1$ for some $j$, then

(a) $K_{3} \subseteq G$,

(b) $G$ is not properly edge-colored, and

(c) $c \leqslant 1 / 2$.

Proof. Since $p+1 \geqslant 5$, at least three vertices of $H$ have positive color degree in $B_{j}$. Now Lemma 2.1 requires that there be one vertex $w$ such that $\hat{d}_{B_{j}}(w)=2$, while $\hat{d}_{B_{j}}\left(w^{\prime}\right)=1$ for each other vertex $w^{\prime}$ in $V(H)$. Now $\left\{u_{j}, v_{j}, w\right\}$ induces a triangle in $G$. Let $\lambda_{1}$ and $\lambda_{2}$ be the colors on $u_{j} w$ and $v_{j} w$, respectively. Partition $V(H)-\{w\}$ into two sets by letting $U=N_{B_{j}}\left(u_{j}\right)-\{w\}$ and $V=N_{B_{j}}\left(v_{j}\right)-\{w\}$. By the maximality of $M$, all edges joining $u_{j}$ to $U$ have color $\lambda_{2}$, and all edges joining $v_{j}$ to $V$ have color $\lambda_{1}$. If $U$ and $V$ are both nonempty, then replacing $u_{j} v_{j}$ with edges to each yields a larger rainbow matching in $G$. Hence $U$ or $V$ is empty and the other has size $p-1$. Now $G$ is not properly edge-colored and either $\hat{d}_{A}\left(u_{j}\right) \leqslant 2$ or $\hat{d}_{A}\left(v_{j}\right) \leqslant 2$. By $(1), 2 c+1 \leqslant 2$ and $c \leqslant 1 / 2$.

\section{Proof of the Main Results}

Theorem 1.1. Every edge-colored graph with minimum color degree $k$ has a rainbow matching of size at least $\lfloor k / 2\rfloor$.

Proof. In the previous notation, the maximum size of a rainbow matching is $k / 2-c$, and $p \geqslant 2 c+2$. Thus $p \leqslant 3$ implies $c \leqslant 1 / 2$. If $p \geqslant 4$ and $c \geqslant 1$, then Lemma 2.2(c) yields $\hat{d}_{B}(V(H)) \leqslant \sum_{j=1}^{k / 2-c} \hat{d}_{B_{j}}(V(H)) \leqslant p(k / 2-c)$, which contradicts $(2)$.

Theorem 1.2. Each condition below guarantees that an $n$-vertex edge-colored graph $G$ with minimum color degree $k$ has a rainbow matching of size at least $\lceil k / 2\rceil$.

(a) $n>\frac{3(k-1)}{2}$.

(b) $G$ is triangle-free.

(c) $G$ is properly edge-colored, $G \neq K_{4}$, and $n \neq k+2$.

Proof. If $G$ has no rainbow matching of size $\lceil k / 2\rceil$, then Theorem 1.1 yields $c=1 / 2$ in the earlier notation. Now (3) implies $\hat{d}_{B}(V(H)) \leqslant \sum_{j=1}^{k / 2-1 / 2} \hat{d}_{B_{j}}(V(H)) \leqslant(p+1)(k / 2-1 / 2)$. Combining this with $(2)$ yields $p(k / 2+1 / 2) \leqslant(p+1)(k / 2-1 / 2)$, which simplifies to $p \leqslant(k-1) / 2$. Hence $n \leqslant 3(k-1) / 2$.

If $G$ is a properly edge-colored complete graph other than $K_{4}$, then the result of Li and $\mathrm{Xu}$ [2] suffices. If $G$ is triangle-free or properly edge-colored with at least $k+3$ vertices, then $p \geqslant 4$ and Lemma 2.2 yield $\hat{d}_{B}(V(H)) \leqslant p(k / 2-c)$, which again contradicts $(2)$.

\section{Acknowledgment}

The authors would like to thank the participants in the combinatorics group of the Research Experience for Graduate Students program at the University of Illinois during summer 2009. Their interest and input is greatly appreciated. 


\section{References}

[1] M. Kano and X. Li, Monochromatic and heterochromatic subgraphs in edge-colored graphs - a survey. Graphs Combin. 24 (2008), 237-263.

[2] X. Li and Z. Xu, On the existence of a rainbow 1-factor in proper coloring of $K_{r n}^{(r)}$. arXiv:0711.2847 [math.CO] 19 Nov 2007.

[3] H. J. Ryser, Neuere Probleme der Kombinatorik, in "Vorträge über Kombinatorik Oberwolfach". Mathematisches Forschungsinstitut Oberwolfach, July 1967, 24-29.

[4] G. Wang and H. Li, Heterochromatic matchings in edge-colored graphs. Electron. J. Combin. 15 (2008), Paper \#R138. 